\title{
THE APPLICATION OF CENTRE-MANIFOLD THEORY TO THE EVOLUTION OF SYSTEMS WYHCH VARY SLOWLY IN SPACE
}

\author{
A. J. ROBERTS
}

(Received 31 October 1986; revised 18 May 1987)

\begin{abstract}
In many physical problems, the system tends quickly to a particular structure, which then evolves relatively slowly in space and time. Various methods exist to derive equations describing the slow evolution of the particular structure; for example, the method of multiple scales. However, the resulting equations are typically valid only for a limited range of the parameters. In order to extend the range of validity and to improve the accuracy, correction terms must be found for the equations. Here we describe a procedure, inspired by centre-manifold theory, which provides a systematic approach to calculating a sequence of successively more accurate approximations to the evolution of the principal structure in space and time.

The formal procedure described here raises a number of questions for future research. For example: what sort of error bounds can be obtained, do the approximations converge or are they strictly asymptotic, and what sort of boundary conditions are appropriate in a given problem?
\end{abstract}

\section{Introduction}

Many physical systems evolve quickly towards a state which can be characterised by a small number of dominant modes. The evolution of the whole system can then be described by the relatively slow evolution of these dominant modes. For example, upon assuming that the particle velocities of a monatomic gas evolve towards equilibrium, it is found that the Euler equation governs the evolution of the mean particle velocity (see Vincenti and Kruger [10]). The more-accurate Navier-Stokes equation governing the evolution of the mean particle velocity may be derived by refining this approximation through assuming that the particle velocities are near equilibrium. The dominant mode here is the equilibrium

${ }^{1}$ Department of Applied Mathematics, University of Adelaide, G. P. O. Box 498, Adelaide, S. A. 5001, Australia.

(C) Copyright Australian Mathematical Society 1988, Serial-fee code 0334-2700/88 
distribution of particle velocities, which is permitted to evolve over length and time scales which are long when compared to molecular length and time scales.

For another example, consider a wavemaker generating a train of water waves on water. Attached to the wavemaker are a number of transients which decay exponentially with distance from the wavemaker, leaving just a uniform train of waves to propagate away as the dominant mode. However, water waves are unstable to the Benjamin-Feir instability, and so over a long length-scale the amplitude of the waves evolves. The application of the procedure described in this paper to this problem of slowly-varying waves is developed in a forthcoming paper [9].

Centre-manifold theory has been developed to produce descriptions of the evolution of such dominant long-lasting modes. Carr [3], Chapter 1 in particular, is an excellent introduction to the theory. The theory states that if the reference state of the system has a number of zero eigenvalues, say $n$, and that if all the other eigenvalues are negative, then the system evolves exponentially quickly towards an $n$-dimensional centre manifold. The system then evolves relatively slowly on the centre manifold according to the evolution of $n$ amplitude functions, for which differential equations can be derived.

The practical calculation of a description of the evolution on a centre manifold of finite dimension has been described by Coullet and Spiegel [4], for thermohaline convection which has a two-dimensional centre manifold, by Roberts [8], for a simple model system with a one-dimensional centre manifold, and by Arneodo et al [1], for triple convection which has a three-dimensional centre manifold. The veracity of the asymptotic analysis has been verified in this last case by Arneodo and Thoul [2] through some direct numerical simulations. Of course, many other perturbation methods have been developed to give similar asymptotic descriptions of a system's evolution; see the discussion in Coullet and Spiegel [4] for examples. However, these methods lack the theoretical background provided by centre-manifold theory. Also (except for the method of reconstitution, see Roberts [6] and [7]), they typically provide only a leading approximation; successive refinement of the approximation is either not possible or extremely tedious to obtain.

The outstanding case of interest is when the centre manifold is of infinite dimension. This case is most often seen as an amplitude evolving in time which is also a function of a space coordinate $x$; the centre manifold then being a function space. More generally, the amplitude could be an evolving vector-valued function of more than one space dimension. Some examples of asymptotic theories which, in essence, involve just such an infinite-dimensional centre manifold are shallow-water theory, convection with the heat flux prescribed on the boundaries, one-dimensional models of rivers, slowly varying waves (Roberts [9]), and gas dynamics. 
Here we extend the procedure of Coullet and Spiegel [4] to a case of an infinitedimensional centre manifold. The procedure is developed through a sequence of simple systems of equations, successive systems introducing new details of the procedure. The basic system of equations is derived from a model of solute transport in soil, the two components in (2.1) representing the advection by water around the soil particles and the advection through the soil particles, together with an exchange term. The basic approximation to be employed is that the solution is slowly varying in the space coordinate $x$; that is, the small parameter in the problem is $\partial / \partial x$. The amplitude of the solution is then a function of $x$, and we want to find a description of its evolution in time.

In Section 2 we consider a linear problem which is transformed so that centremanifold theory (as described by Carr [3]) can be directly applied. In Section 3, the same problem is approached via the new procedure. The results are the same, which gives confidence that the procedure works. However, note that an infinitedimensional centre-manifold theorem has apparently not yet been proved. What is presented here is just a formal procedure which is a generalisation of the method of Coullet and Spiegel [4] for finite-dimensional centre manifolds. Proofs of existence and estimates of error bounds are not yet available; further work needs to be done.

We then introduce nonlinearity into the problem. In Section 4, the nonlinearity is specially designed so that the only approximation made is that the solution is slowly varying in $x$. This problem is unusual among nonlinear problems, in that normally a small amplitude has to be assumed as well. Thus in Section 5 and Section 6 we change the nonlinearity and investigate the typical approach of assuming that both the amplitude and the dependence upon $x$ are small.

One difficulty which arises is in deciding what sort of boundary condition to apply when using the results of this analysis. The problem is that the evolution on the centre manifold is often govened by a differential equation of higher differential order than the original equations; for example, compare (2.19) with the original system (2.1). It would appear that near a boundary the system may (although not necessarily) be held away from the state of near equilibrium expressed by the centre manifold. Thus some sort of transition layer between the boundary and the interior of the domain may be needed, and this is discussed further at the end of Section 2.

\section{The spectral evolution of a two component flow}

Here we begin investigating a problem with the property that its solutions typically evolve to be slowly varying in space. In this section, we analyse it via 
a spectral approach which enables a more-or-less rigorous application of centremanifold theory. The problem is to study the solutions of the coupled pair of linear differential equations

$$
\begin{aligned}
\partial a / \partial t+v_{a} \partial a / \partial x & =(-a+b) / 2, \\
\partial b / \partial t+v_{b} \partial b / \partial x & =(+a-b) / 2,
\end{aligned}
$$

where $v_{a}$ and $v_{b}$ are the velocities with which interacting components $a$ and $b$, respectively, are being transported in a one-dimensional continuum. No matter what the initial conditions are, the solutions behave like this. Over a long enough time the interaction, represented on the right-hand-side of equation (2.1), tends to equalise the values of $a$ and $b$ at any point. The difference between the advection velocities, $v_{a}$ and $v_{b}$, combined with this interaction, tends to smooth the dependence of $a$ and $b$ upon space $x$. Thus the solutions are eventually slowly varying in space, and this process is precisely what centre-manifold theory quantifies.

Equation (2.1) may be written equivalently in terms of $\mathrm{U}=(a, b)^{T}$ as

$$
\partial \mathbf{U} / \partial t=L \mathbf{U}-M \partial \mathbf{U} / \partial x,
$$

where

$$
L=\frac{1}{2}\left(\begin{array}{cc}
-1 & 1 \\
1 & -1
\end{array}\right) \text { and } M=\left(\begin{array}{cc}
v_{a} & 0 \\
0 & v_{b}
\end{array}\right)
$$

Upon taking the spatial Fourier transform of equation (2.2), using $\kappa$ to denote the wavenumber and ${ }^{\wedge}$ to denote transformed quantities, we can write down that

$$
\partial \hat{\mathbf{U}} / \partial t=L \hat{\mathbf{U}}-i \kappa M \hat{\mathbf{U}} .
$$

The utility of the Fourier transform in this linear problem can now be seen: the evolution equation (2.4) for each wavenumber component has become completely uncoupled from other wavenumbers. Thus the evolution of each wavenumber will be treated separately, and then combined together at the end.

To apply the centre-manifold theory to (2.4), we now change the basis of the $\hat{U}$-plane to a basis which clearly separates the long term structure of the solutions from the short term. Introducing the two new unknowns

$$
c=a+b \text { and } d=a-b
$$

we expect $d$ to become very small and $c$ to evolve slowly in space. After making this substitution, (2.4) becomes

$$
\begin{aligned}
& \partial \hat{c} / \partial t=0-i \kappa(\bar{v} \hat{c}+\tilde{v} \hat{d}), \\
& \partial \hat{d} / \partial t=-\hat{d}-i \kappa(\tilde{v} \hat{c}+\bar{v} \hat{d}),
\end{aligned}
$$


where

$$
\bar{v}=\left(v_{a}+v_{b}\right) / 2 \text { and } \tilde{v}=\left(v_{a}-v_{b}\right) / 2 .
$$

Observe that $\bar{v}$ is the mean advection velocity of the two components, while $\tilde{v}$ gives the difference from this mean of the actual velocities. To apply centremanifold theory to (2.6), the linear part of the right-hand-side must have one or more zero eigenvalues corresponding to the dominant long lasting modes, and the rest of the eigenvalues must be negative. Since we consider $\kappa$ to be consiant, we can arrange this by supplementing (2.6) with

$$
d \kappa / d t=0,
$$

and temporarily consider $\kappa$ to be a dependent variable. Then the terms involving $\kappa$ in (2.6) become nonlinear terms, and hence the linear part of the combined system (2.6) and (2.8) has two zero eigenvalues and one negative eigenvalue (-1).

Applying centre-manifold theory (Carr [3]) to (2.6) and (2.8), we know that there exists a centre manifold

$$
\hat{d}=h(\hat{c}, \kappa) \quad \text { such that } h(0,0)=\partial h / \partial \hat{c}(0,0)=\partial h / \partial \kappa(0,0)=0,
$$

on which $\hat{c}$ and $\kappa$ evolve according to

$$
d \hat{c} / d t=g(\hat{c}, \kappa) \text { and } d \kappa / d t=0,
$$

where $g$ and $h$ are yet to be determined. Furthermore, we know that the centre manifold (2.9) is approached exponentially-quickly in time. However, note that the concept of a centre manifold is only clear-cut for small $\hat{c}$ and small $\kappa$; that is, for weak variations in $x$. Although there is typically a unique centre manifold in some finite domain, the separation of the system into an exponential approach and a slow evolution is only valid near the origin. See Figure 1 in Roberts [6] for an illustration of this.

Upon substituting (2.10) into the first equation of (2.6), we find that

$$
g(\hat{c}, \kappa)=-i \kappa[\bar{v} \hat{c}+\tilde{v} h(\hat{c}, \kappa)] .
$$

Upon substituting the ansatz (2.9) and (2.10) into the second equation of (2.6), and using the above expression for $g$, we find that $h$ should satisfy the following equation

$$
-i \kappa \frac{\partial h}{\partial \hat{c}}(\bar{v} \hat{c}+\tilde{v} h)=-h-i \kappa(\tilde{v} \hat{c}+\bar{v} h),
$$

together with the conditions on $h$ at the origin. Now, as this problem is originally linear, the exact description of the centre manifold can be found to be

$$
h(\hat{c}, \kappa)=\frac{i}{2 \tilde{v} \kappa}\left(-1+\sqrt{1-4 \tilde{v}^{2} \kappa^{2}}\right) \hat{c}
$$

on which $\hat{c}$ evolves according to

$$
\partial \hat{c} / \partial t=g(\hat{c}, \kappa)=\left[-i \bar{v} \kappa-\frac{1}{2}+\frac{1}{2} \sqrt{1-4 \tilde{v}^{2} \kappa^{2}}\right] \hat{c} .
$$


However, being able to find an exact solution is not typical. In general, the centre manifold can only be described near the origin and only asymptotically.

Approximations to the centre manifold can be easily found by iteration. Rearrange (2.12) into the iteration scheme:

$$
\begin{aligned}
& h_{0}=0, \\
& h_{n+1}=-i \tilde{v} \kappa \hat{c}-i \bar{v} \kappa h_{n}+i \kappa \frac{\partial h_{n}}{\partial \hat{c}}\left(\bar{v} \hat{c}+\tilde{v} h_{n}\right)
\end{aligned}
$$

(a form which is analogous to those used in later sections). We find, for example, that

$$
h_{2}=-i \tilde{v} \kappa \hat{c}-i \tilde{v}^{3} \kappa^{3} \hat{c}
$$

This represents, of course, the first two terms of the Taylor series expansion of (2.13). Another theorem from centre-manifold theory says that if (2.12) is satisfied to some order of accuracy when the independent variables $\hat{c}, \kappa \rightarrow 0$, then the centre manifold is approximated to the same order of accuracy. Thus

$$
\hat{d}=h(\hat{c}, \kappa)=-i \tilde{v} \kappa \hat{c}-i \tilde{v}^{3} \kappa^{3} \hat{c}+O\left(|(\hat{c}, \kappa)|^{5}\right),
$$

on which $\hat{c}$ evolves according to

$$
\partial \hat{c} / \partial t=g(\hat{c}, \kappa)=-i \bar{v} \kappa \hat{c}-\tilde{v}^{2} \kappa^{2} \hat{c}-\tilde{v}^{4} \kappa^{4} \hat{c}+O\left(|(\hat{c}, \kappa)|^{6}\right),
$$

and where $\kappa$ is constant, according to (2.10).

To return to spatial dependence, we now take the inverse Fourier transform of (2.16) and (2.17) to obtain

$$
d=-\tilde{v} \frac{\partial c}{\partial x}+\tilde{v}^{3} \frac{\partial^{3} c}{\partial x^{3}}+\cdots
$$

where $c$ evolves according to

$$
\partial c / \partial t=-\bar{v} \frac{\partial c}{\partial x}+\tilde{v}^{2} \frac{\partial^{2} c}{\partial x^{2}}-\tilde{v}^{4} \frac{\partial^{4} c}{\partial x^{4}}+\cdots .
$$

Thus upon inverting (2.5) we find that the centre manifold is approximately described by

$$
\mathrm{U}=\left[\begin{array}{l}
a \\
b
\end{array}\right]=\left[\begin{array}{l}
1 / 2 \\
1 / 2
\end{array}\right] c-\frac{\tilde{v}}{2}\left[\begin{array}{c}
1 \\
-1
\end{array}\right] \frac{\partial c}{\partial x}+\frac{\tilde{v}^{3}}{2}\left[\begin{array}{c}
1 \\
-1
\end{array}\right] \frac{\partial^{3} c}{\partial x^{3}}+\cdots,
$$

where the evolution on the centre manifold is described by (2.19).

As predicted at the start of this section, we have observed that solutions to (2.1) evolve exponentially-quickly to the centre manifold (2.20), a state where the two components are nearly equal. Furthermore, the evolution on the centre manifold is governed by (2.19), which indicates that spatial variations in $a$ and $b$ are primarily advected with the mean velocity $\bar{v}$, and also diffuse depending upon the difference in the velocities, $\tilde{v}$; in other problems this is known as shear diffusion. However, in more interesting nonlinear problems the process of taking 
the Fourier transform and changing the basis is not one which is easily managed. A more direct approach is desirable, and this is developed in the next section.

It is apparent from (2.19) that the equation describing the evolution on the centre manifold is of higher differential order than the original problem (2.1). The question then is: what sort of boundary conditions should be applied on a spatial boundary? The difficulty which arises is really due to the fact that asymptotically-slow variations in space and a finite spatial extent do not nit together; the limit $\kappa \rightarrow 0$ implicitly needs an infinite spatial extent to be realised. However, many problems involving the slowly-varying approximation (for example, the evolution of water waves) are discussed without reference to spatial boundaries, the boundary conditions being left open. Other problems (for example, convection near the onset of motion) use periodic boundary conditions which are just as easy to express for the centre manifold as for the full problem.

However, some problems do involve a nontrivial boundary (for example: the shallow-water approximation near a beach). In these cases, the description of the centre manifold supplies boundary conditions for the evolution on the centre manifold. For example, if in the above problem the condition that $a=a_{0}$ and $b=b_{0}$ is given at some point $x=x_{0}$ then (2.20) supplies the conditions

$$
c=a_{0}+b_{0} \text { and }-\tilde{v} \frac{\partial c}{\partial x}+\tilde{v}^{3} \frac{\partial^{3} c}{\partial x^{3}}=a_{0}-b_{0} \quad \text { at } x=x_{0},
$$

for the evolution equation (2.19). However, some boundary conditions will be incompatible with the description of the centre manifold, such a boundary condition acts to hold the system away from the centre manifold. Since the centre manifold is stable, we expect that there must then be a region near the boundary in which an exponential transition is made to the centre manifold. Such boundary layers are familiar in many mathematical approximations.

Moreover, some equations of unquestioned practical value have similar problems. For example, the Euler equation is used widely in fluid mechanics at high Reynolds numbers, the presence of viscous boundary layers being of little importance to the main flow, unless flow separation occurs. Even the more accurate Navier-Stokes equation, which needs more boundary conditions to accommodate its higher differential order, can fail at a boundary, namely near a moving contact line or the tip of a crack. It would seem that the difficulties with the centre manifold near a boundary are surmountable, but need more attention.

\section{The spatial evolution of a two-component flow}

Here we re-examine the problem (2.1), with the aim of deriving the description of the centre manifold (2.20) and the evolution on it (2.19) via a more direct approach than that employed in Section 2. We follow the procedure outlined in 
Coullet and Spiegel [4], but generalised, via concepts taken from the theory of calculus of variations, to an infinite-dimensional centre manifold (assuming that it exists and is sufficiently well-behaved).

We first have to define some measure of the local "amplitude" of solutions to (2.1). Recognising that solutions typically have the two components $a$ and $b$ nearly equal, an obvious choice for a local amplitude is

$$
A(x, t)=a+b=U_{1}+U_{2} .
$$

Almost any other definition will do, but this one has the advantage that the results of this section can be directly compared with those of Section 2 .

We then suppose that the centre manifold can be described by

$$
\mathrm{U}(x, t)=\mathrm{V}[A ; x]
$$

where the evolution on the centre manifold is described by

$$
\partial A / \partial t=G[A ; x],
$$

where square brackets are used to denote a functional dependence in $x$ upon the argument $A$. For example: $G[A ; x]$ indicates that $G$ depends upon $A, \partial A / \partial x$, $\partial^{2} A / \partial x^{2}, \ldots ;$ thus we may write

$$
G[A ; x]=G\left(A, A^{\prime}, A^{\prime \prime}, \ldots\right),
$$

where we shall use' to denote differentiation with respect to $x$. In a simplistic fashion we consider functions of $x$ to be a vector space and choose the functions $\left(x-x_{0}\right)^{n} / n$ ! (where $x_{0}$ is any point) to be a basis for this space. Thus the independent components of $A$ with respect to this basis are the coefficients of the Taylor series of $A$ about $x_{0}$, namely $\left(A, A^{\prime}, A^{\prime \prime}, \ldots\right)$.

To substitute the ansatz (3.2) and (3.3) into the governing differential equation (2.2), we have to be careful about differentiation, just as in the calculus of variations. We use partials to denote the total derivative with respect to a given variable, while a subscript will denote differentiation with respect to that subscript symbol. Thus, assuming (3.2) and (3.3) and that $A$ is a differentiable function of $(x, t)$, we observe

$$
\partial \mathbf{U} / \partial t=\mathbf{V}_{A} G+\mathbf{V}_{A^{\prime}} \frac{\partial G}{\partial x}+\mathbf{V}_{A^{\prime \prime}} \frac{\partial^{2} G}{\partial x^{2}}+\cdots
$$

where

$$
\partial G / \partial x=G_{A} A^{\prime}+G_{A^{\prime}} A^{\prime \prime}+\cdots,
$$

and so on for higher-order derivatives. Upon substituting (3.2) and (3.3) into (2.2), we can then derive that $\mathrm{V}$ and $G$ must satisfy

$$
L \mathrm{~V}=\left(\mathrm{V}_{A} G+\mathrm{V}_{A^{\prime}} \frac{\partial G}{\partial x}+\mathrm{V}_{A^{\prime \prime}} \frac{\partial^{2} G}{\partial x^{2}}+\cdots\right)+M\left(\mathrm{~V}_{A} A^{\prime}+\mathrm{V}_{A^{\prime}} A^{\prime \prime}+\cdots\right)
$$


We have arranged the equation into this form so that the left-hand-side, the linear $L \mathrm{~V}$, equates to the "small" terms on the right-hand-side (recognising that the right-hand-side represents only the slowly-varying terms $\partial \mathbf{U} / \partial t$ and $\partial \mathbf{U} / \partial x$ ). To an initial approximation, the right-hand-side terms can be neglected and thus $\mathrm{V}$ lies approximately in the null space of $L$, proportional to $(1,1)^{T}$, and is some function of $x$ and $t$. This observation is what we now develop systematically.

Given the typical situation where the exact descriptioun of $G[A ; x]$ and $\vee[A ; x]$ cannot be found, we then wish to find asymptotic expressions for them. In principle (and necessarily so in later sections), the expressions for $G$ and $\mathrm{V}$ are multinomials in the independent variables (the indeterminates) $A, A^{\prime}, A^{\prime \prime}, \ldots$. However, as this problem is linear, the multinomials must be linear, and hence we can take a short cut by assuming this now. Thus we try the linear form

$$
\mathrm{V} \sim \sum_{k=0}^{\infty} \mathrm{V}^{k} A^{(k)} \text { and } G \sim \sum_{k=0}^{\infty} G^{k} A^{(k)}
$$

where the superscript on $G$ and $\mathbf{V}$ denotes a member of a family rather than exponentiation, and where $A^{(k)}$ is the symbol for the $k$ th derivative of $A$ with respect to $x$. Upon substituting (3.5) into (3.4) and equating the coefficients of the independent variables, $A, A^{\prime}, A^{\prime \prime}, \ldots$, we find the sequence of equations

$$
\begin{aligned}
& L \mathbf{V}^{0}=G^{0} \mathbf{V}^{0}, \\
& L \mathbf{V}^{k}=M \mathbf{V}^{k-1}+\sum_{l=0}^{k} G^{l} \mathbf{V}^{k-l}, \quad k=1,2, \ldots
\end{aligned}
$$

The $k=0$ equation is just an eigenproblem for the eigenvalue $G^{0}$ and corresponding eigenvector $\mathbf{V}^{0}$ of $L$. The eigenvalues of $L$ are 0 and -1 . The slow evolution along the centre manifold can only be deduced from the presence of the 0 eigenvalue; the eigenvalue -1 is irrelevant as it only describes the exponential approach to the centre manifold. $\mathbf{V}^{\mathbf{0}}$ is a corresponding eigenvector and is thus proportional to $(1,1)^{T}$. To fix the particular multiple, we use the definition (3.1) of the local "amplitude" $A$. From (3.1), (3.2) and (3.5) we know

$$
A=U_{1}+U_{2}=\sum_{k=0}^{\infty}\left(V_{1}^{k}+V_{2}^{k}\right) A^{(k)},
$$

and so by equating the coefficients of the independent variables we want

$$
V_{1}^{k}+V_{2}^{k}=\left\{\begin{array}{ll}
1, & k=0 \\
0, & k=1,2, \ldots
\end{array},\right.
$$

where $V_{1}^{k}$ and $V_{2}^{k}$ are the two components of $\mathrm{V}^{k}$. Thus the solution of the $k=0$ equations is

$$
G^{0}=0 \text { and } \quad \mathbf{V}^{0}=\left[\begin{array}{l}
1 / 2 \\
1 / 2
\end{array}\right]
$$


which is just the basic approximation that the two components $a$ and $b$ eventually equalise.

The $k=1$ equation of (3.6) reduces to

$$
L V^{1}=\left[\begin{array}{l}
v_{a} / 2 \\
v_{b} / 2
\end{array}\right]+G^{1}\left[\begin{array}{l}
1 / 2 \\
1 / 2
\end{array}\right]
$$

For general values of the as-yet-unknown $G^{1}$, this equation cannot be solved as $L$ is singular; only if $G^{1}$ is chosen appropriately can we solve it for $\mathrm{V}^{1}$. To find $G^{\mathbf{1}}$ we find first the left-eigenvector;

$$
z=\left[\begin{array}{ll}
1 & 1
\end{array}\right],
$$

of $L$ corresponding to the eigenvalue $G^{0}=0$; in more general situations $\mathbf{z}$ would be in the null space of the adjoint of $L$. Upon multiplying (3.9) on the left by $z$, we deduce

$$
G^{1}=\left(v_{a}+v_{b}\right) / 2=-\bar{v},
$$

which is often known as a solvability condition. Upon using the amplitude equations (3.7), the solution for $\mathrm{V}^{1}$ becomes unique and is

$$
\mathbf{V}^{1}=\left[\begin{array}{c}
\left(v_{b}-v_{a}\right) / 4 \\
\left(v_{a}-v_{b}\right) / 4
\end{array}\right]=-\frac{\tilde{v}}{2}\left[\begin{array}{c}
1 \\
-1
\end{array}\right]
$$

In an exactly similar manner to the above, the equations (3.6) and (3.7) can be solved for $k=2,3, \ldots$ in succession to give

$$
\begin{gathered}
G^{2}=\tilde{v}^{2} \quad \text { and } \quad \mathrm{V}^{2}=0, \\
G^{3}=0 \quad \text { and } \quad \mathrm{V}^{3}=\frac{\tilde{v}^{3}}{2}\left[\begin{array}{c}
1 \\
-1
\end{array}\right], \\
G^{4}=-\tilde{v}^{4} \quad \text { and } \quad \mathrm{V}^{4}=0,
\end{gathered}
$$

etc. Thus the centre manifold (3.2) is described by

$$
\mathrm{U} \sim\left[\begin{array}{l}
1 / 2 \\
1 / 2
\end{array}\right] A-\frac{\tilde{v}}{2}\left[\begin{array}{c}
1 \\
-1
\end{array}\right] A^{\prime}+\frac{\tilde{v}^{3}}{2}\left[\begin{array}{c}
1 \\
-1
\end{array}\right] A^{\prime \prime \prime},
$$

on which the evolution takes place according to (3.3) which is

$$
\partial A / \partial t \sim-\bar{v} A^{\prime}+\tilde{v}^{2} A^{\prime \prime}-\tilde{v}^{4} A^{\prime \prime \prime \prime} .
$$

This is precisely the same approximation, (2.19) and (2.20), as derived in Section 2.

This approach to describing the long-time evolution of solutions to (2.1) is apparently valid provided the high-order derivatives of $A$ are sufficiently small; it is essentially a perturbation expansion in $\partial / \partial x$. Although no error bounds can be obtained at the moment, the derivation of this same approximation in Section 2 suggests that it does indeed become invalid if the spatial variations are too rapid. 
In Section 2, the exact description in Fourier space of the centre manifold and its evolution, (2.13) and (2.14), exhibits a square-root singularity at a wavenumber of $\kappa=1 / 2 \tilde{v}$. Thus the asymptotic descriptions must surely become invalid if the solutions obtained involve significant components at wavenumbers higher than this.

The actual procedure in finding the $G^{k}$ and $\mathrm{V}^{k}$ is very familiar to anyone who has done a multiple-scale or slowly-varying asymptotic analysis. The main difference is that this procedure has a different theoretical background, that of centre-manifold theory. It is this different background which enables a number of artifices in conventional slowly-varying analyses to be avoided. For example: there is no need to shift to a moving frame of reference as is often done to get a significant approximation (here the fact that the components move at the mean advection velocity appears naturally and does not affect the rest of the analysis); there is no need to introduce super-slow and extra-super-slow space-time scales to refine the description of the ultimate evolution (here the extra terms in the refinement appear naturally); there is no redundant algebra which the technique of reconstitution involves.

All these points indicate that this is a very powerful and widely applicable technique. Further ramifications and developments of the method are illustrated in the next two sections.

\section{The spatial evolution of a special nonlinear problem}

We now consider a modified version of the govening equations (2.1). Nonlinear terms are introduced, but in such a fashion that the only approximation which needs to be made is that the spatial derivatives are small. Nonlinear problems typically require the additional assumption that the amplitude is also small; such a problem is investigated in the next two sections.

Additionally we also permit the material properties, or parameters, of the system to vary in space. As a particular example, we have suppose that the advection velocities $v_{a}$ and $v_{b}$ are given, slowly-varying functions of $x$. Other parameters of the system could also vary slowly in space (namely the exchange $L$ and the nonlinearity c), but to keep the results relatively simple we consider them constant. Variations in time of the parameters can also be accomodated (see Roberts [9]), but in essence it would introduce a second small parameter into the problem, and so needs a double asymptotic sum (see the next section) rather than the single asymptotic sum developed here.

Consider the problem

$$
\frac{\partial \mathrm{U}}{\partial t}=L \mathrm{U}-\frac{\partial}{\partial x} M \mathrm{U}+\mathrm{c}\left(U_{1}^{2}-U_{2}^{2}\right)
$$


where $L$ and $M$ are given by (2.3) and where

$$
c=\left[\begin{array}{l}
1 \\
1
\end{array}\right]
$$

There is an exact solution to (4.1), namely

$$
\mathrm{U}=\left[\begin{array}{l}
1 / 2 \\
1 / 2
\end{array}\right] A
$$

where $A$ is any constant. It is the presence of this exact solution to the nonlinear problem which enables the assumption that the solution is varying slowly in space to be the only assumption made.

We now assume that $\partial / \partial x$ is a "small quantity". Consequently, a term appearing in an equation will be called order $k$ if it contains precisely $k$ spatial derivatives. For example: some terms of order 2 are

$$
A^{\prime \prime}, A^{2}, A A^{\prime \prime}, A A^{2}, v_{a} A^{\prime \prime}, v_{b}^{\prime} v_{a} A^{\prime}, v_{a}^{\prime \prime} A^{2} .
$$

Following Coullet \& Spiegel [4] more closely now, we substitute the centremanifold ansatz, namely (3.2) and (3.3), into the governing (4.1) and seek the asymptotic approximation

$$
\mathrm{V} \sim \sum_{k=0}^{\infty} \mathbf{V}^{k}[A ; x] \text { and } G \sim \sum_{k=1}^{\infty} G^{k}[A ; x],
$$

where $G^{k}$ and $\mathrm{V}^{k}$ contain all the terms of order $k$. Note that $G^{k}[A ; x]$ is of order $k$ if $G^{k}[A ; X / \varepsilon]=\varepsilon^{k} G^{k}[A ; X]$ for all $\varepsilon$; in this test, $X$ is recognised to be analogous to a long-space scale. (Note: we have anticipated that $G^{\mathbf{0}}=0$, which was found to be necessary in the previous section in order to describe the centre manifold.) Upon equating all terms of the same order together in the equations we find that

$$
\begin{aligned}
& L \mathrm{~V}^{0}=-\mathrm{c} N^{0} \\
& L \mathrm{~V}^{k}=\frac{\partial}{\partial x} M \mathrm{~V}^{k-1}-\mathrm{c} N^{k}+\sum_{\ell=1}^{k} \sum_{p=0}^{k-\ell}\left(\mathrm{V}_{A_{(p)}}^{k-\ell} \frac{\partial^{p} G^{\ell}}{\partial x^{p}}\right), \quad k=1,2, \ldots,
\end{aligned}
$$

where the nonlinear terms are represented by

$$
N^{k}=\sum_{\ell=0}^{k}\left(V_{1}^{\ell} V_{1}^{k-\ell}-V_{2}^{\ell} V_{2}^{k-\ell}\right),
$$

in which $V_{1}$ and $V_{2}$ are the two components of $V$. Observe in (4.3) that the sum over $p$ (that is, the differentiation with respect to the derivatives of $A$ ) is truncated to $p=k-\ell$ as $\mathrm{V}^{k-\ell}$ can have no more than $k-\ell$ spatial derivatives in it. The definition of the local amplitude must also be considered. Upon 
substituting (4.2) into (3.1) and equating all terms of the same order we find that

$$
V_{1}^{k}+V_{2}^{k}= \begin{cases}A, & k=0 \\ 0, & k>0\end{cases}
$$

We now proceed to solve (4.3) and (4.5) sequentially.

The $k=0$ equation is

$$
L V^{0}=-c\left[\left(V_{1}^{0}\right)^{2}-\left(V_{2}^{0}\right)^{2}\right]
$$

which, together with (4.5), has the solution

$$
\mathrm{V}^{0}=\left[\begin{array}{l}
1 / 2 \\
1 / 2
\end{array}\right] A \text {. }
$$

Once again, this just represents the fact that over long enough times the two components tend to equalise.

After substituting the solution for $\mathrm{V}^{0}$ into (4.3), the $k=1$ equation becomes

$$
(L+\mathrm{c}[1-1] A) \mathrm{V}^{1}=\frac{1}{2}\left[\begin{array}{c}
v_{a} A^{\prime}+v_{a}^{\prime} A \\
v_{b} A^{\prime}+v_{b}^{\prime} A
\end{array}\right]+\left[\begin{array}{l}
1 / 2 \\
1 / 2
\end{array}\right] G^{1} .
$$

This equation has some rather unusual features. The unknowns $\mathrm{V}^{1}$ and $G^{1}$ are multinomials in the indeterminates $A$ and $A^{\prime}$. Thus the equation is like a polynomial one, and a solution can only be found if, in some sense, the factor $L+\mathbf{c}[1-1] A$ "divides" the right-hand-side. It turns out that we can be assured of this only if the two components of $c$ are equal; hence the choice for $c$. Upon multiplying (4.8) on the left by the left-eigenvector $\mathrm{z}$, just as in the previous section, we deduce that

$$
G^{1}+\bar{v} A^{\prime}+\bar{v}^{\prime} A=2[1-1] A \mathbf{V}^{1},
$$

which reflects an unusual feature in this type of problem in that $G^{\mathbf{1}}$ is finally determined only after $V^{1}$ is, rather than before. After substituting (4.9) into equation (4.8) it reduces to

$$
L V^{1}=\frac{1}{2}\left[\begin{array}{c}
1 \\
-1
\end{array}\right]\left(\tilde{v} A^{\prime}+\tilde{v}^{\prime} A\right)
$$

which, together with (4.5), has the solution

$$
\mathbf{V}^{1}=-\frac{1}{2}\left[\begin{array}{c}
1 \\
-1
\end{array}\right]\left(\tilde{v} A^{\prime}+\tilde{v}^{\prime} A\right)
$$

and consequently

$$
G^{1}=-\bar{v} A^{\prime}-2 \tilde{v} A A^{\prime}-\bar{v}^{\prime} A-2 \tilde{v}^{\prime} A^{2} .
$$

The first nontrivial approximation to the centre manifold is thus

$$
\mathbf{U} \sim\left[\begin{array}{c}
1 / 2 \\
1 / 2
\end{array}\right] A-\frac{1}{2}\left[\begin{array}{c}
1 \\
-1
\end{array}\right](\tilde{v} A)^{\prime}
$$


on which the quantities evolve according to

$$
\partial A / \partial t \sim-(\bar{v} A)^{\prime}-2 A(\tilde{v} A)^{\prime} .
$$

The above approximation may be refined further by calculating more terms of the asymptotic expansion. The general scheme needed is no more complicated that that described above for the $k=1$ case, the only complication being that the number of terms increases. For example, the solution for order- 2 quantities is

$$
\begin{gathered}
\mathbf{V}^{2}=-\left[\begin{array}{c}
1 \\
-1
\end{array}\right]\left\{\left(\tilde{v} A(\tilde{v} A)^{\prime}\right)^{\prime}+\frac{1}{2}\left(\tilde{v} \bar{v}^{\prime} A-\tilde{v}^{\prime} \bar{v} A\right)^{\prime}\right\}, \\
G^{2}=\left(\tilde{v}(\tilde{v} A)^{\prime}\right)^{\prime}-4 A\left(\tilde{v} A(\tilde{v} A)^{\prime}\right)^{\prime}+2 A\left(\tilde{v}^{\prime} \bar{v} A-\tilde{v} \bar{v}^{\prime} A\right)^{\prime} .
\end{gathered}
$$

Thus a second approximation to the centre manifold and the evolution on it is given by

$$
\begin{gathered}
\mathbf{U} \sim\left[\begin{array}{c}
1 / 2 \\
1 / 2
\end{array}\right] A-\left[\begin{array}{c}
1 \\
-1
\end{array}\right]\left\{\frac{1}{2}(\tilde{v} A)^{\prime}+\left(\tilde{v} A(\tilde{v} A)^{\prime}\right)^{\prime}+\frac{1}{2}\left(\tilde{v} \bar{v}^{\prime} A-\tilde{v}^{\prime} \bar{v} A\right)^{\prime}\right\}, \\
\partial A / \partial t \sim-(\bar{v} A)^{\prime}-2 A(\tilde{v} A)^{\prime}+\left(\tilde{v}(\tilde{v} A)^{\prime}\right)^{\prime}-4 A\left(\tilde{v} A(\tilde{v} A)^{\prime}\right)^{\prime}+2 A\left(\tilde{v}^{\prime} \bar{v} A-\tilde{v} \bar{v}^{\prime} A\right)^{\prime} .
\end{gathered}
$$

This nonlinear problem is rather special in that the only approximation made was that the solution and the parameters are slowly-varying in space; consequently it involved some unusual features. Most nonlinear problems will also require that the solution be of small amplitude. This additional assumption removes the above unusual features and makes the whole process very familiar; it just has a different theoretical background.

\section{Spatial evolution with a typical nonlinearity}

We now turn to another modified version of (2.1). A more-or-less arbitrary nonlinear term is introduced which necessitates the asymptotic description of the centre manifold to involve two small parameters. We need to assume that both $A$ and $\partial / \partial x$ are small. This type of problem, where the amplitude is assumed small and the length scale is assumed long, is typical of many interesting problems.

Consider the problem

$$
\partial \mathrm{U} / \partial t=L \mathrm{U}-M \partial \mathrm{U} / \partial x+\mathrm{c}\left(U_{1}+U_{2}\right)^{2}
$$

where $L$ and $M$ are given by (2.3) and where

$$
c=\left[\begin{array}{l}
1 \\
1
\end{array}\right] \bar{c}+\left[\begin{array}{c}
1 \\
-1
\end{array}\right] \tilde{c}
$$

For simplicity we consider that the parameters of the system are constant in both space and time. The modification needed to include variations in $x$ is trivial, 
and just leads to greater complexity in the results. Slow variations in time of the parameters can also be included, but would introduce a third "small quantity", namely $\partial / \partial t$. We omit such variation here (but see Roberts [9]).

We now assume that both $\partial / \partial x$ and $A$ are "small quantities". Consequently a term appearing in an equation will be called $\operatorname{order}(k, m)$ if it contains precisely $k$ spatial derivatives and precisely $m$ amplitude factors $A$. For example: all the terms of order $(3,4)$ are

$$
A^{3} A^{\prime \prime \prime}, A^{2} A^{\prime} A^{\prime \prime} \text {, and } A\left(A^{\prime}\right)^{3} .
$$

We now substitute the centre-manifold ansatz, namely (3.2) and (3.3), into (5.1) and seek the asymptotic description

$$
\mathrm{V} \sim \sum_{k=0}^{\infty} \sum_{m=1}^{\infty} \mathbf{V}^{k, m}[A ; x] \text { and } G \sim \sum_{k=0}^{\infty} \sum_{m=1}^{\infty} G^{k, m}[A ; x],
$$

where $G^{k, m}$ and $\mathrm{V}^{k, m}$ contain all the terms of order $(k, m)$. Note that a functional $f[A ; x]$ is of order $(k, m)$ if $f[\delta B ; X / \varepsilon]=\varepsilon^{k} \delta^{m} f[B ; X]$, for all $\varepsilon$ and $\delta$.

Upon equating all terms of the same order together we find that

$$
\begin{array}{r}
L \mathrm{~V}^{k, m}=M \frac{\partial \mathbf{V}^{k-1, m}}{\partial x}-\mathbf{c} N^{k, m}+\sum_{\ell=0}^{k} \sum_{n=1}^{m} \sum_{p=0}^{k-\ell} \mathrm{V}_{A^{(p)}}^{k-\ell, m-n+1} \frac{\partial^{p} G^{\ell, n}}{\partial x^{p}} \\
\quad \text { for all } k \text { and } m,
\end{array}
$$

where $\mathbf{V}^{-1, m}=\mathbf{0}$ and where the nonlinear terms are represented by

$$
N^{k, m}=\sum_{\ell=0}^{k} \sum_{n=1}^{m-1}\left(V_{1}^{\ell, n}+V_{2}^{\ell, n}\right)\left(V_{1}^{k-\ell, m-n}+V_{2}^{k-\ell, m-n}\right) .
$$

The definition of the local amplitude must also be considered. Upon substituting (5.2) into (3.1) and equating terms of the same order we find that

$$
V_{1}^{k, m}+V_{2}^{k, m}=\left\{\begin{array}{ll}
A, & (k, m)=(0,1) \\
0, & \text { otherwise }
\end{array} .\right.
$$

We can now proceed to solve the doubly-infinite family of equations (5.3) and (5.5), for all $k$ and $m$. Any sequential order can be used, provided that all quantities of order $(\ell, n)$ such that $\ell \leq k$ and $n \leq m$ are known, except $(k, m)$ itself, before quantities of order $(k, m)$ are calculated.

The crucial equation and its solution, from which all the other equations and solutions follow, is the one of order $(0,1)$. It is

$$
L \mathrm{~V}^{0,1}=\mathrm{V}_{A}^{0,1} G^{0,1}
$$

which should be solved in conjunction with the appropriate version of (5.5). This is, just as in Section 3, a sort of eigen-problem. The eigenvector which begins the 
description of the centre manifold is the one corresponding to the zero eigenvalue, thus

$$
G^{0,1}=0 \quad \text { and } \quad \mathbf{V}^{0,1}=\left[\begin{array}{l}
1 / 2 \\
1 / 2
\end{array}\right] A .
$$

This is, as always, the basic approximate solution to this problem.

We now proceed to calculate higher-order quantities. First we shall arbitrarily calculate quantities of order $(0,2)$ and $(0,3)$. We find that $(5.3)$ with $k=0$ and $m=2$ becomes

$$
L \mathrm{~V}^{0,2}=\left[\begin{array}{l}
1 / 2 \\
1 / 2
\end{array}\right] G^{0,2}-\mathrm{c} A^{2}
$$

Once again, this should be regarded as a multinomial equation with the indeterminates $A, A^{\prime}, A^{\prime \prime}, \ldots$ However, unlike Section 4 there is never any difficulty in doing this, as the unknown $\mathbf{V}^{\mathbf{0}, 2}$ is multiplied simply by a "constant" $L$. Thus we can always find the solution by "dividing" through by $L$. In a now-familiar process, we recognise that $L$ is singular and so this equation can only be solved if $G^{0,2}$ is chosen appropriately. Upon multiplying on the left by the left-eigenvector of $L$, namely $\mathbf{z}$, we find $G^{0,2}$ and then, in conjunction with (5.5), solve for $\mathbf{V}^{0,2}$ to find that

$$
G^{0,2}=2 \bar{c} A^{2} \quad \text { and } \quad \mathrm{V}^{0,2}=\tilde{c}\left[\begin{array}{c}
1 \\
-1
\end{array}\right] A^{2} .
$$

The order- $(0,3)$ version of $(5.3)$ is

$$
L \mathrm{~V}^{0,3}=\left[\begin{array}{l}
1 / 2 \\
1 / 2
\end{array}\right] G^{0,3}+4 \tilde{c} \bar{c}\left[\begin{array}{c}
1 \\
-1
\end{array}\right] A^{3} .
$$

We can solve this in conjunction with (5.5) to give

$$
G^{0,3}=0 \quad \text { and } \quad \mathbf{V}^{0,3}=-4 \tilde{c} \bar{c}\left[\begin{array}{c}
1 \\
-1
\end{array}\right] A^{3} .
$$

Equations (5.7), (5.8) and (5.9) provide an approximate description of the evolution of the system, namely that the centre manifold is

$$
\mathrm{U} \sim\left[\begin{array}{c}
1 / 2 \\
1 / 2
\end{array}\right] A+\left[\begin{array}{c}
1 \\
-1
\end{array}\right]\left(\tilde{c} A^{2}-4 \tilde{c} \bar{c} A^{3}\right)
$$

on which the system evolves according to

$$
\partial A / \partial t \sim 2 \bar{c} A^{2} .
$$

In principle we could calculate an arbitrary number of terms in this asymptotic expansion. However, this description is not adequate if any significant spatial variations are present. All it does is describe the nonlinear evolution of the system when every quantity is constant in $x$.

To take the spatial variations into account we have to calculate quantities involving $\partial / \partial x$. Thus, here we calculate quantities of order $(1,1)$ and $(2,1)$ 
starting from the initial approximation (5.7). The equation corresponding to $k=1$ and $m=1$ is

$$
L V^{1,1}=\left[\begin{array}{l}
1 / 2 \\
1 / 2
\end{array}\right] G^{1,1}+\frac{1}{2}\left[\begin{array}{l}
v_{a} \\
v_{b}
\end{array}\right] A^{\prime},
$$

which, together with (5.5), has the solution

$$
G^{1,1}=-\bar{v} A ; \quad \text { and } \quad \mathrm{V}^{1,1}=-\frac{\tilde{v}}{2}\left[\begin{array}{c}
1 \\
-1
\end{array}\right] A^{\prime} .
$$

The equation corresponding to $k=2$ and $m=1$ is

$$
L V^{2,1}=\left[\begin{array}{l}
1 / 2 \\
1 / 2
\end{array}\right] G^{2,1}-\frac{\tilde{v}^{2}}{2}\left[\begin{array}{l}
1 \\
1
\end{array}\right] A^{\prime \prime},
$$

which, in conjunction with (5.5), has the solution

$$
G^{2,1}=\tilde{v}^{2} A^{\prime \prime} \text { and } \mathrm{V}^{2,1}=\mathbf{0} .
$$

We can put (5.7), (5.10) and (5.11) together to give another approximate description of the centre manifold to be

$$
\mathrm{U} \sim\left[\begin{array}{l}
1 / 2 \\
1 / 2
\end{array}\right]-\frac{\tilde{v}}{2}\left[\begin{array}{c}
1 \\
-1
\end{array}\right] A^{\prime},
$$

on which the amplitude $A$ evolves according to

$$
\partial A / \partial t \sim-\bar{v} A^{\prime}+\tilde{v}^{2} A^{\prime \prime}
$$

In principle we could also extend this sequence to arbitrarily many terms. If this were done we would obtain precisely the same asymptotic expansion as found in Section 3 (compare the above with (3.16) and (3.17)); this is because this sequence is always linear in the amplitude (as $m=1$ ). Although this sequence involves spatial variations, it takes no account of the nonlinear interaction terms present in the original equations (5.1).

To derive a version of the centre manifold and the evolution on it which takes account of the effects of both nonlinear interactions and also variations in $x$, we could justifiably combine (5.7) through to (5.11). However, it is usual to include terms which represent an action which is a mixture of the nonlinearity and the spatial variations. We can do this by calculating terms of order $(k, m)$ where $k>0$ and $m>1$; here we calculate just the term of order $(1,2)$. This term's equation is

$$
L \mathrm{~V}^{1,2}=\left[\begin{array}{l}
1 / 2 \\
1 / 2
\end{array}\right] G^{1,2}+2 \tilde{c} \tilde{v}\left[\begin{array}{l}
1 \\
1
\end{array}\right] A A^{\prime}-2 \tilde{v} \bar{c}\left[\begin{array}{c}
1 \\
-1
\end{array}\right] A A^{\prime}
$$

Upon solving this we find that

$$
G^{1,2}=-4 \tilde{c} \tilde{v} A A^{\prime} \quad \text { and } \quad V^{1,2}=2 \tilde{v} \bar{c}\left[\begin{array}{c}
1 \\
-1
\end{array}\right] A A^{\prime} .
$$


Another consistent approximation, for example, is then to combine terms of order $(k, m)$ where $0 \leq k \leq 1$ and $1 \leq m \leq 2$ (here ignoring the fact that we have found the terms of order $(0,3)$ and order $(2,1))$. Combining $(5.7),(5.8)$, (5.10) and (5.12) we would then find that the centre manifold is

$$
\mathbf{U} \sim\left[\begin{array}{l}
1 / 2 \\
1 / 2
\end{array}\right] A+\left[\begin{array}{c}
1 \\
-1
\end{array}\right]\left(-\frac{1}{2} \tilde{v} A^{\prime}+\tilde{c} A^{2}+2 \tilde{v} \bar{c} A A^{\prime}\right)
$$

on which the evolution is

$$
\partial A / \partial t \sim-\bar{v} A^{\prime}+2 \bar{c} A^{2}-4 \tilde{c} \tilde{v} A A^{\prime} .
$$

This is another example of a description which involves both the nonlinear effects and the spatial variations. Its new feature is that it possesses a term which contains both effects mixed in.

The above is an example of the general scheme for applying a formal centre manifold ansatz to nonlinear problems in which the centre manifold is of infinite dimension. It can be readily generalised to cope with more than one independent "amplitude", and more than one spatial dimension for the centre manifold. However, the plethora of subscripts and superscripts becomes compounded in more-involved problems. The next section describes briefly the consequences of a common approach which simplifies the appearance of the process.

\section{A comment on the combined slowly-varying weakly-nonlinear assumption}

In asymptotic expansions with two or more small parameters, as there are in Section 5, it is often the case that the two (or more) small parameters are linked together. For example, we could have assumed that $\partial / \partial x$ is of the same order as $A$, which is of the same order as some small number, say $\varepsilon$. Exactly this will be done here and, instead of having a doubly-infinite family of equations to solve, we obtain a singly-infinite family as in the earlier sections. A further simplification is that the algebraic form of the equations has considerably fewer terms, the extra terms being hidden in the unknowns of the asymptotic analysis. The price of all this simplification is the loss of flexibility in the choice of retained quantities; a benefit is that it is easier to automate the whole process.

We reconsider equation (5.1) with constant parameters, and substitute the centre-manifold ansatz, (3.2) and (3.3), and seek an asymptotic description of the form

$$
\mathrm{V} \sim \sum_{k=1}^{\infty} \mathrm{V}^{k}[A ; x] \text { and } G \sim \sum_{k=1}^{\infty} G^{k}[A ; x],
$$

where $G^{k}$ and $\mathrm{V}^{k}$ contain all the terms of order $k$. In this section we say that an functional expression $f[A ; x]$ is order $k$ if $f[\varepsilon B ; X / \varepsilon]=\varepsilon^{k} f[B ; X]$, for all $\varepsilon$. 
For example, all the terms of order 4 are

$$
A^{\prime \prime \prime}, A A^{\prime \prime},\left(A^{\prime}\right)^{2}, A^{2} A^{\prime} \text {, and } A^{4} \text {. }
$$

This definition then implicitly links the amplitude $A$ and the spatial variations $\partial / \partial x$ together to both be of the same magnitude, namely $\varepsilon$. A useful consequence, relating the definitions of order, is that a term of order $(k, m)$ in Section 5 becomes a term of order $k+m$ in this section. Upon substituting (6.1) and equating all terms of the same order together we find that

$$
\begin{aligned}
& L \mathbf{V}^{1}=\mathbf{V}_{A}^{1} G^{1}, \\
& L \mathbf{V}^{k}=M \frac{\partial \mathbf{V}^{k-1}}{\partial x}-\mathbf{c} N^{k}+\sum_{\ell=1}^{k} \sum_{p=0}^{k-\ell} \mathbf{V}_{A(p)}^{k-\ell+1} \frac{\partial^{p} G^{\ell}}{\partial x^{p}}, \quad k=2,3, \ldots,
\end{aligned}
$$

where the nonlinear terms are represented by

$$
N^{k}=\sum_{\ell=1}^{k-1}\left(V_{1}^{\ell}+V_{2}^{\ell}\right)\left(V_{1}^{k-\ell}+V_{2}^{k-\ell}\right) .
$$

The sequence of equations given by (6.2) must be solved in conjunction with the definition of the local amplitude, which in this section requires

$$
V_{1}^{k}+V_{2}^{k}= \begin{cases}A, & k=1, \\ 0, & k=2,3, \ldots .\end{cases}
$$

The procedure is now the same as in Section 5, except that quantities must be found in a strict sequence; first $k=1$, then $k=2$, then $k=3$, etc.

However, all the terms which appear in the equations and their solutions in Section 5 appear precisely the same here. A term of order $(k, m)$ in Section 5 occurs correspondingly as a term of order $k+m$ in this section. Thus, from the results of Section 5,

$$
G^{k}=\sum_{\ell=1}^{k} G^{k-\ell, \ell} \quad \text { and } \quad \mathbf{V}^{k}=\sum_{\ell=1}^{k} \mathbf{V}^{k-\ell, \ell},
$$

and the solutions can be written down to be

$$
\begin{aligned}
& G^{1}=0 \quad \text { and } \quad \mathrm{V}^{1}=\left[\begin{array}{c}
1 / 2 \\
1 / 2
\end{array}\right] A \\
& G^{2}=-\bar{v} A^{\prime}+2 \bar{c} A^{2} \quad \text { and } \quad \mathrm{V}^{2}=\left[\begin{array}{c}
1 \\
-1
\end{array}\right]\left(\tilde{c} A^{2}-\frac{\tilde{v}}{2} A^{\prime}\right) \\
& G^{3}=\tilde{v}^{2} A^{\prime \prime}-4 \tilde{c} \tilde{v} A A^{\prime} \quad \text { and } \quad \mathrm{V}^{3}=\left[\begin{array}{c}
1 \\
-1
\end{array}\right]\left(2 \tilde{v} \bar{c} A A^{\prime}-4 \tilde{c} \bar{c} A^{3}\right),
\end{aligned}
$$

and so on. Hence, upon truncating the sums in (6.1) to the first three terms, we find that the centre manifold is given by

$$
\mathrm{U} \sim\left[\begin{array}{c}
1 / 2 \\
1 / 2
\end{array}\right] A+\left[\begin{array}{c}
1 \\
-1
\end{array}\right]\left(\tilde{c} A^{2}-\frac{\tilde{v}}{2} A^{\prime}+2 \tilde{v} \bar{c} A A^{\prime}-4 \tilde{c} \bar{c} A^{3}\right)
$$


on which the solution evolves according to

$$
\partial A / \partial t \sim-\bar{v} A^{\prime}+2 \bar{c} A^{2}+\tilde{v}^{2} A^{\prime \prime}-4 \tilde{c} \tilde{v} A A^{\prime} .
$$

The reason that all this works is that the expressions are considered to be multinomials in the independent indeterminates $A, A^{\prime}, A^{\prime \prime}, \ldots$ Thus at each order in the calculation the terms are always distinguishable by the different products of these indeterminates in each term. This also means that, given the results of Section 5 , we can very easily vary the relative magnitudes of the amplitude and the spatial variations. For example: suppose that the amplitude $A$ is of the size of $\varepsilon$ but that spatial variations are much smaller (but not negligible), say of the size of $\varepsilon^{2}$; then quantities appearing at order $(k, m)$ in Section 5 would then appear at order $2 k+m$. Hence, assuming a connection between the relative magnitudes of the various small parameters in a problem, and then truncating the asymptotic sum (which is often done) is equivalent to drawing a line in the plane (or space) of exponents, here the $(k, m)$-plane, and only including those terms which lie on one side of the line.

\section{Conclusion}

The formal procedure proposed in this paper is simply a generalisation of that of Coullet and Spiegel [4] to asymptotic problems which are conventionally known as slowly varying in space and time. It generalises their procedure (also explained in Roberts [8]), which deals with a finite-dimensional centre manifold, to the case of an infinite-dimensional manifold. Instead of predicting the evolution of a vector, we predict the evolution of a function of space from which the detailed approximate solution can be found.

This procedure has many advantages over other methods which produce similar approximations. The procedure is completely mechanistic once the initial approximation has been identified; there is no need to go into long heuristic arguments in the derivation of successive approximations. There is no need for artifices such as transforming to a moving frame of reference, as is done, for example, in the derivation of the nonlinear Schrödinger equation for slowly-varying waves. There is no need to introduce extra super-slow space or time scales to produce successive approximations, as is needed in the method of multiple scales (see section 3.5 in Jeffrey and Kawahara [5] for example). There is no redundant algebra as is needed in the method of reconstitution (see Roberts [6] and [7]). Also there is no need, although it is often convenient, to make assumptions about the relative magnitude of different "small" effects in the problem (for example, the relative magnitude of nonlinearity and variations in space); thus it clarifies the asymptotic analysis when two or more small parameters are involved. 
The last big advantage of this procedure is that it is inspired by centremanifold theory. Thus we can be reasonably confident that the proofs currently in existence for finite-dimensional centre manifolds (see Carr [3]) can be generalised to apply to these infinite-dimensional centre manifolds. However, a lot of research remains to be done on the sort of problems to which this procedure is valid, to determine how to obtain error bounds, and how to handle boundaries in space.

\section{Acknowledgements}

I wish to acknowledge the support given by the Australian Research Grant Scheme and a University Research Grant. I thank Glen Walker and Mike Eastwood for making me re-think these types of problems.

\section{References}

[1] A. Arneodo, P. H. Coullet and E. A. Spiegel, "The dynamics of triple convection", Geophys. Astrophys. 31, (1985), 1-48.

[2] A. Arneodo and O. Thoul, "Direct numerical simulation of a triple convection problem versus the normal-form prediction", Phys. Lett. A 109, (1985), 367-347.

[3] J. Carr, Applications of centre manifold theory, Applied Mathematical Sciences, 35, (Springer-Verlag 1981).

[4] P. H. Coullet and E. A. Spiegel, "Amplitude equations for systems with competing instabilities", SIAM J. Appl. Math. 43 (1983), 776-821.

[5] A. Jeffrey and T. Kawahara, Asymptotic methods in nonlinear wave theory, (Pitman, 1982).

[6] A. J. Roberts, "An introduction to the technique of reconstitution", SIAM J. Math. Anal. 16 (1985), 1241-1257.

[7] A. J. Roberts, "An analysis of near-marginal, mildly penetrative convection with heat flux prescribed on the boundaries", J. Fluid Mech. 158 (1985), 71-93.

[8] A. J. Roberts, "Simple examples of the derivation of amplitude equations for systems of equations possessing bifurcations", J. Austral. Math. Soc. B 27 (1985), 48-65.

[9] A. J. Roberts, "The application of centre manifold theory to the evolution of slowlyvarying waves", in preparation (1987).

[10] W. G. Vincenti and C. H. Kruger, Introduction to Physical Gas Dynamics, (John Wiley, New York 1965). 